\title{
The Solar Dynamics Observatory (SDO)
}

\author{
W. Dean Pesnell • B.J. Thompson · P.C. Chamberlin
}

Received: 16 June 2011 / Accepted: 7 August 2011 / Published online: 18 October 2011

(C) The Author(s) 2011. This article is published with open access at Springerlink.com

\begin{abstract}
The Solar Dynamics Observatory (SDO) was launched on 11 February 2010 at 15:23 UT from Kennedy Space Center aboard an Atlas V 401 (AV-021) launch vehicle. A series of apogee-motor firings lifted SDO from an initial geosynchronous transfer orbit into a circular geosynchronous orbit inclined by $28^{\circ}$ about the longitude of the SDO-dedicated ground station in New Mexico. SDO began returning science data on 1 May 2010. SDO is the first space-weather mission in NASA's Living With a Star (LWS) Program. SDO's main goal is to understand, driving toward a predictive capability, those solar variations that influence life on Earth and humanity's technological systems. The SDO science investigations will determine how the Sun's magnetic field is generated and structured, how this stored magnetic energy is released into the heliosphere and geospace as the solar wind, energetic particles, and variations in the solar irradiance. Insights gained from SDO investigations will also lead to an increased understanding of the role that solar variability plays in changes in Earth's atmospheric chemistry and climate. The SDO mission includes three scientific investigations (the Atmospheric Imaging Assembly (AIA), Extreme Ultraviolet Variability Experiment (EVE), and Helioseismic and Magnetic Imager (HMI)), a spacecraft bus, and a dedicated ground station to handle the telemetry. The Goddard Space Flight Center built and will operate the spacecraft during its planned five-year mission life; this includes: commanding the spacecraft, receiving the science data, and forwarding that data to the science teams. The science investigations teams at Stanford University, Lockheed Martin Solar Astrophysics Laboratory (LMSAL), and University of Colorado Laboratory for Atmospheric and Space Physics (LASP) will process, analyze, distribute, and archive the science data. We will describe the building of SDO and the science that it will provide to NASA.
\end{abstract}

The Solar Dynamics Observatory Guest Editors: W. Dean Pesnell, Phillip C. Chamberlin, and Barbara J. Thompson

W.D. Pesnell $(\bowtie) \cdot$ B.J. Thompson · P.C. Chamberlin

NASA Goddard Space Flight Center, Greenbelt, MD, USA

e-mail: william.d.pesnell@nasa.gov

B.J. Thompson

e-mail: barbara.j.thompson@nasa.gov

P.C. Chamberlin

e-mail: phillip.c.chamberlin@nasa.gov 
Keywords SDO $\cdot$ Solar cycle $\cdot$ Helioseismology $\cdot$ Coronal $\cdot$ Space weather

\section{Preface: Living with a Star and the Solar Dynamics Observatory}

The goal of NASA's Living With a Star (LWS) Program is to provide the scientific understanding needed to address those aspects of heliophysics science that may affect life and society, where heliophysics is the study of the Sun and how it influences and drives changes in all objects within its reach. The ultimate goal is to develop the ability to predict conditions at Earth and in the interplanetary medium due to the Sun's ever-changing output, a subject called space weather.

LWS is designed to give us a better understanding of the causes of space weather, whose effects can disable satellites, cause power-grid failures, and disrupt global positioning system and other communications signals. The LWS program includes coordinated strategic missions, missions of opportunity, a targeted research and technology program, a spaceenvironment testbed flight opportunity, and partnerships with other agencies and nations. Each LWS mission is designed to answer specific science questions needed to understand the interconnected systems that impact us.

Radiation Belt Storm Probes (RBSP), the second LWS mission, will be launched in 2012 to study the acceleration to high energies of electrons in the Earth's radiation belts. Balloon Array for Radiation Belt Relativistic Electron Losses (BARREL) is a multiple-balloon investigation of the Earth's radiation belts that will fly from Antarctic stations during the southern Summers of 2012 and 2013. Two solar missions, Solar Probe Plus and Solar Orbiter (a joint mission with ESA), are planned for launch later this decade. They will study the coronal heating and solar-wind acceleration problems from platforms flying close to the Sun and out of the Ecliptic.

LWS is a part of the International Living With a Star (ILWS) Program, an organization bringing together researchers and space-weather operators to further develop our ability to understand and predict space weather.

\section{Introduction}

The Solar Dynamics Observatory (SDO) is designed to provide the data and scientific understanding necessary to predict solar activity, from anticipating whether flares and CMEs will occur the next day to the level of solar activity in future solar cycles. Monitoring the topology of magnetic fields as they are formed within the Sun itself through the outer atmosphere, or corona, by high-resolution images may provide the precursors for predicting flares and CMEs. A key insight that SDO hopes to provide is the topological configuration that is needed to drive reconnection and reorganization in these built-up and stressed magnetic fields - the energy release and source that drives the solar eruptive events.

Longer timescales require a knowledge of how magnetic field is transported, amplified, and destroyed inside the Sun and ultimately ejected from the interior - the solar dynamo. Between these limiting timescales another goal of SDO is to predict where and when active regions will emerge, how those magnetic fields erupt and decay, and how the host of other phenomena related to the solar magnetic field come and go.

Solar Cycle 24 started in December 2008 and is predicted to rise to a below-average peak in 2013. This affords an excellent laboratory for SDO. All previous space-based missions with significant duration sampled Solar Cycles 20-23, which had above-average levels of 
activity. The latter half of the 20th century is estimated to have the highest levels of solar activity in the past 10000 years (Solanki et al., 2004). Solar Cycle 23 was well-measured by the still-operating SOHO and Hinode missions, with STEREO starting at the beginning of the extended solar-minimum period.

Two instruments on SDO concentrate on the energy radiated in the extreme ultraviolet. Solar Extreme Ultraviolet (EUV: $1-122 \mathrm{~nm}$ ) photons are the dominant cooling radiation of the solar corona and are also the dominant source of heating for Earth's upper atmosphere. When the Sun is active, EUV emissions can rise and fall by factors of hundreds (and Xrays by factors of thousands) in just a matter of seconds. These surges heat the Earth's upper atmosphere, inflating it and increasing the drag on satellites. EUV photons also can break the bonds of atmospheric atoms and molecules, creating a layer of ions that alters and sometimes severely disturbs radio communications and global positioning system navigation.

SDO will also measure variations inside the Sun, providing the information needed to understand the internal motions that generate the magnetic field, and then the magnetic field as it emerges through the solar surface. The combination of the various measurements allows SDO to study the lifecycle of the solar magnetic field.

To achieve its goals SDO will transmit approximately 150000 high-resolution full-Sun images and 9000 EUV spectra, or 1.5 terabyte of science data, to the ground each day during the prime mission. This will be converted into images, Dopplergrams, magnetograms, and spectra. Over the five-year prime mission, SDO will return roughly 3-4 petabytes of raw data.

The rapid cadence and improved spatial resolution of the SDO instruments is already providing new science results. Images from AIA quickly showed how the global magnetic field of the Sun must relax after even a modest flare and filament (Schrijver and Title, 2011). The low level of flaring during the first year of SDO science operations allowed the discovery that the energy released in many flares is poorly estimated by the GOES X-ray radiometers (Woods et al., 2011b). Energy is radiated at longer wavelengths and for a longer time a significant finding for people modeling the response of the terrestrial atmosphere to flares.

\section{History}

The Solar Dynamics Observatory is a flagship mission that represents a substantial enhancement of capabilities demonstrated by earlier NASA missions and other science projects. Those earlier measurements revealed new details about the solar interior and the mechanisms driving the solar dynamo. Unlike the line-of-sight (LOS) magnetographs flown on earlier missions, SDO carries a full-disk imaging vector magnetograph with a data cadence sufficient to see changes in the magnetic-field strength and direction that could be related to flares or CMEs. Another needed capability was rapid-cadence images of the solar corona with wavelength coverage to span temperatures from the chromosphere $(10000 \mathrm{~K})$ to flares $\left(2 \times 10^{7} \mathrm{~K}\right)$. Full-disk images with spatial resolution of $\approx 1^{\prime \prime}$ are needed to both resolve known spatial scales and to sample the variations across the disk. The solar images from Yohkoh, SOHO, and Skylab were used by solar physicists to understand solar activity and by space-weather forecasters to monitor solar activity. Additional bandpasses to better estimate the plasma temperature and higher cadence were both required to understand the response of the corona to the injected magnetic field.

One direct connection between solar activity and the Earth is through the emissions at extreme ultraviolet wavelengths $(1-122 \mathrm{~nm})$. The utility of these measurements was demonstrated by the SEE instrument on the TIMED satellite (Woods et al., 2005). An instrument 
with higher duty cycle and cadence would measure the total-energy content in flares while also providing the data to calibrate the coronal images and measuring the radiant energy output of the Sun that creates the ionosphere.

Enhancing the helioseismic and LOS magnetic-field capabilities demonstrated by the MDI instrument on SOHO and the ground-based GONG network was part of a proposal to NASA entitled SONAR (or Hale). This mission had advanced sufficiently far toward realization that it was moved to the LWS program and revised to become SDO. The initial organization of the LWS program by the LWS Science Architecture Team occurred in parallel with the development of SDO.

A Science Definition Team for SDO was formed in November 2000 with David Hathaway (NASA/MSFC) as the chair and Barbara Thompson (NASA/GSFC) as the Study Scientist. The team met several times until July 2001, when the SDT Report (Hathaway et al., 2001) that codified all of the measurement and science requirements was released. This report formed the basis for the NASA Announcement of Opportunity AO 02-OSS-01 soliciting science investigations, which was released 18 January 2002.

The HMI and EVE Science Investigation Teams (SITs) were selected on 19 August 2002 while the AIA SIT was selected on 7 November 2003. The observatory was built and completed testing by September 2008, when delays in the Atlas V launcher line caused it to be left in storage until June 2009. At that time SDO was moved to Florida for launch preparations and launched on 11 February 2010. Science operations began on 1 May 2010 and will continue for the baseline lifetime of five years. There are no consumables on the spacecraft that will limit the useful life of the mission. It is estimated that over 100 years of propellant is left in the tanks.

SDO has several features unique to a NASA science mission:

i) A sustained high rate of science-data production and the movement of those data within the spacecraft. This requires reliable electronics that are robust against radiation-induced effects while maintaining timing tolerances better than $1 \mathrm{~ns}$.

ii) The continuous downlink of science data, using a highly automated data pipeline to process the data for immediate use by the space-weather community as well as SDO science investigations.

iii) The extremely accurate pointing and stability required of the spacecraft to allow registration of successive images.

iv) The long (five years) mission life with high-duty cycle instruments.

To date all of these features but the fourth are working and only the long lifetime has yet to be proven.

Because SDO was designed to measure high-resolution data for a five-year mission, the science data resolve the eruptive events, such as flares and coronal mass ejections, while building a well-characterized database for long-term studies. By combining SDO data with those of other missions, such as SOHO, TRACE, Hinode, and TIMED, we will be able to construct a picture of the Sun over an entire 22-year magnetic cycle. It is these long-term research projects that should solve the puzzle of the solar cycle and the space weather it creates at Earth.

SDO was built by a team made up of the Goddard Space Flight Center, Stanford University, in Palo Alto, California, the Lockheed Martin Solar Astrophysics Laboratory (LMSAL), also in Palo Alto, California, and the Laboratory for Atmospheric and Space Physics (LASP), University of Colorado in Boulder, Colorado. Goddard was responsible for designing and building the spacecraft and many of the components of the spacecraft. The other team members are the Science Investigation Teams, who are responsible for designing and 
Figure 1 The SDO spacecraft with the instruments, high-gain antennas, and solar arrays highlighted. The thrusters and main engine are located on the opposite face of the satellite.

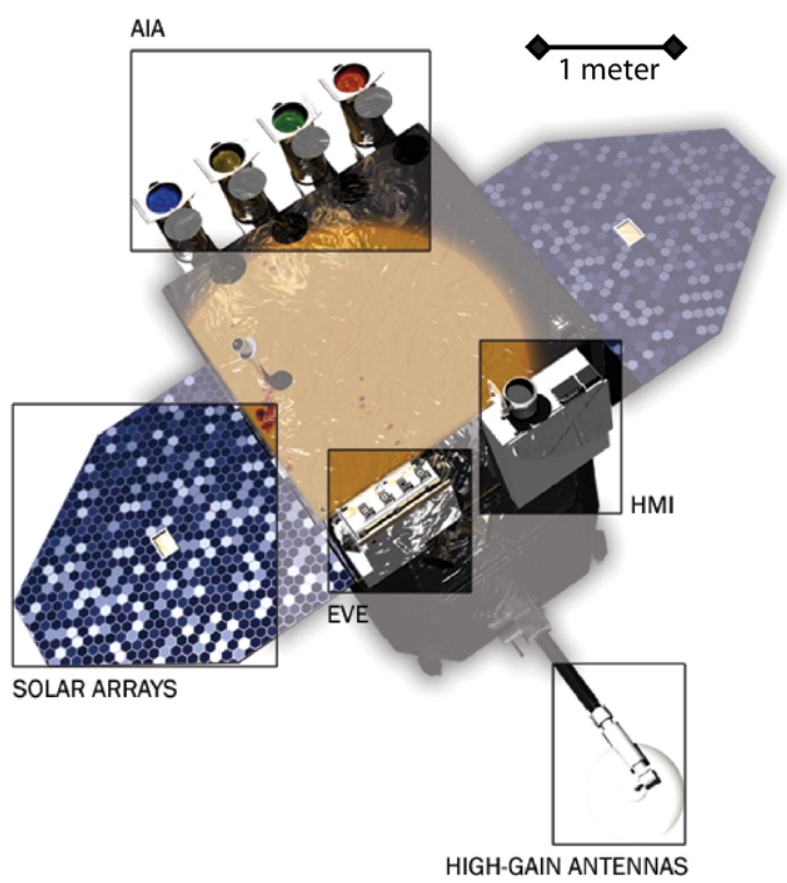

building their instrument, for running the instruments after launch, and performing science investigations related to the data produced by the instruments.

\section{The Spacecraft Summary}

The SDO spacecraft is shown in Figure 1. A summary of relevant information is:

Satellite: Three-axis stabilized and fully redundant spacecraft.

Duration: Five-year science mission and sufficient propellant to operate for an additional five years.

Mass: Total mass at launch was $3000 \mathrm{~kg}$ : instruments $300 \mathrm{~kg}$, spacecraft $1300 \mathrm{~kg}$, and fuel $1400 \mathrm{~kg}$.

Dimensions: The overall length of the spacecraft along the Sun-pointing axis is $4.7 \mathrm{~m}$, and each side is $2.2 \mathrm{~m}$. The span of the spacecraft is $6.1 \mathrm{~m}$ along the extended solar panels and $6.0 \mathrm{~m}$ along the deployed high-gain antennas.

Solar Array: The $6.6 \mathrm{~m}^{2}$ of solar panels produce $1500 \mathrm{~W}$ of power with an estimated efficiency of $16 \%$ after five years on orbit. The "homeplate" shape prevents the solar panel from blocking the high-gain antennas.

High-gain Antennas: The high-gain antennas rotate once each orbit to track the ground station.

Maximum Downlink Rate: The spacecraft has a continuous, high-rate science-data downlink rate of $150 \mathrm{Mbps}$ at a Ka-Band frequency of about $26 \mathrm{GHz}$; includes $130 \mathrm{Mbps}$ of data and $20 \mathrm{Mbps}$ of encoding overhead.

Mission Operations Center: Located at Goddard Space Flight Center in Maryland.

SDO Ground Station: Located at White Sands Complex in New Mexico. 
Table 1 The SDO Level 1 science requirements.

Scientific Questions

What mechanisms drive the quasi-periodic 11-year cycle of solar activity?

How is active region magnetic flux synthesized, concentrated, and dispersed across the solar surface?

How does magnetic reconnection on small scales reorganize the large-scale field topology and current systems, and how significant is it in heating the corona and accelerating the solar wind?

Where do the observed variations in the Sun's extreme ultraviolet spectral irradiance arise, and how do they relate to the magnetic-activity cycles?

What magnetic-field configurations lead to the coronal mass ejections, filament eruptions, and flares that produce energetic particles and radiation?

Can the structure and dynamics of the solar wind near Earth be determined from the magnetic-field configuration and atmospheric structure near the solar surface?

When will activity occur, and is it possible to make accurate and reliable forecasts of space weather and climate?

Table 2 The instrument science objectives.

Gauge the energy from solar activity that is input into the Earth's atmosphere and near-Earth space

Probe the dynamics of the near-surface shear layer of the Sun to observe the local strong-flux regions before they reach the photosphere

Probe the internal processes that govern the solar cycle

Observe the initiation and progression of dynamic processes in the chromosphere and corona to understand their connection to the Sun's magnetic field.

Table 3 SDO measurement objectives.

Make measurements over a significant portion of a solar cycle to capture the solar variations that exist on all time scales from seconds (solar eruptive events) to months (active region evolution, solar rotation) to years (solar cycle)

Measure the extreme ultraviolet spectral irradiance of the Sun at a rapid cadence

Measure the Doppler shifts due to oscillation velocities over the entire visible disk

Make high-resolution measurements of the longitudinal and vector magnetic field over the entire visible disk

Make images of the chromosphere and inner corona at several temperatures at a rapid cadence.

\section{Science Goals}

The scientific goals of the SDO project, which were first posed in the SDO Science Definition Team report (Hathaway et al., 2001), are to improve our understanding of the seven science questions listed in Table 1. The instruments must be designed to meet the instrument science objectives listed in Table 2 using the measurements listed in Table 3. All of the questions are related to developing an understanding of the solar magnetic field. Three of the science questions emphasize predicting solar activity.

To satisfy these science goals, the Science Investigation Teams described next have created specific research topics and goals. 
Table 4 The SDO science investigations.

\begin{tabular}{lll}
\hline Investigation & Abbrev. & Returned Data \\
\hline Heliospheric and Magnetic Imager & HMI & $\begin{array}{l}\text { Full-disk Dopplergrams } \\
\text { Full-disk LOS magnetograms }\end{array}$ \\
& & $\begin{array}{l}\text { Full-disk vector magneograms } \\
\text { Rapid-cadence, full-disk EUV solar images }\end{array}$ \\
Atmospheric Imaging Assembly & AIA & $\begin{array}{l}\text { Rapid-cadence EUV spectral irradiance } \\
\text { Extreme Ultraviolet Variability Experiment }\end{array}$
\end{tabular}

\section{Science Investigation Teams}

The science payload of SDO comprises three instrument suites built by the Science Investigation Teams (SIT). The SITs are summarized in Table 4. The instrument suites are described in detail in other articles in this Topical Issue, so we provide only a brief summary of each.

\subsection{Atmospheric Imaging Assembly}

The Atmospheric Imaging Assembly (AIA) is an array of four telescopes that observes the surface and atmosphere of the Sun. The AIA Principal Investigator is Alan Title of LMSAL. AIA was built to obtain full-disk images of the solar atmosphere with a field of view of at least $40^{\prime}$ and a two-pixel resolution of $1.2^{\prime \prime}$. Filters on the telescopes cover ten different wavelength bands that include seven extreme ultraviolet, two ultraviolet, and one visiblelight band to reveal key aspects of solar activity. The wavelength bands were chosen to yield diagnostics over the range of $6000 \mathrm{~K}$ to $3 \times 10^{6} \mathrm{~K}$. AIA uses multilayer coatings combined with foil filters to isolate the desired spectral bandpasses for each telescope. AIA was built by LMSAL, Palo Alto, California. A detailed description of AIA can be found in Lemen et al. (2011).

\subsection{Extreme Ultraviolet Variability Experiment}

The Extreme ultraviolet Variability Experiment (EVE) measures fluctuations in the Sun's ultraviolet output. Tom Woods of LASP is the Principal Investigator. EVE will measure the solar spectral irradiance in the most variable and unpredictable part of the solar spectrum from 0.1 to $105 \mathrm{~nm}$ as well as $121.6 \mathrm{~nm}$. EVE has three parts: MEGS, ESP, and SAM. MEGS combines grating spectrometers with two CCDs to create images of the spectral irradiance in various wavelength ranges. These individual sections are then combined to give the spectrum between 6.5 to $105 \mathrm{~nm}$ at $0.1 \mathrm{~nm}$ spectral resolution. A silicon photodiode strategically placed inside the MEGS-B channel measures the irradiance of Lyman- $\alpha$ at $121.6 \mathrm{~nm}$, the single brightest line in the EUV. ESP is a series of radiometers placed behind a transmission grating that measures the irradiance in several wavelength bands $(0.1-5.9$, 17.2 - 20.6, 23.1 - 27.6, 28.0 - 31.6, and 34.0 - 38.1 nm). It is similar to the SEM instrument on SOHO. SAM is a pinhole camera used with the MEGS-A CCD to measure individual $\mathrm{X}$-ray photons in the wavelength range $0.1-7 \mathrm{~nm}$.

This design will allow solar scientists to continuously monitor EUV emissions at a tensecond cadence. EVE was built by LASP at the University of Colorado. A detailed description of the EVE instrument and science investigation can be found in Woods et al. (2011a). 


\subsection{Helioseismic and Magnetic Imager}

The Helioseismic and Magnetic Imager (HMI) will map magnetic and velocity fields at the surface of the Sun. A key goal of this experiment is to decipher the physics of the solar magnetic dynamo. The Principal Investigator for HMI is Phil Scherrer of Stanford University. HMI was built by LMSAL, Palo Alto, California. A detailed description of the HMI science investigation is given in Schou et al. (2011).

HMI measures the Doppler shift of the Fe I $617.3 \mathrm{~nm}$ spectral line to determine the photospheric surface velocity over the Sun's entire visible disk. HMI creates a full-disk photospheric velocity measurement (or Dopplergram) every 45 seconds with a two-pixel resolution of $1^{\prime \prime}$, a noise level $\approx 25 \mathrm{~m} \mathrm{~s}^{-1}$, a data recovery of $95 \%$ of the Dopplergrams, and a data completeness of $99 \%$ for each Dopplergram. These Dopplergrams are then used to study the inside of the Sun.

HMI uses the Zeeman effect of the same spectral line to measure the Stokes parameters required to create full-disk longitudinal magnetic-field measurements (LOS magnetogram) and full-disk vector photospheric magnetic-field maps (vector magnetogram). The LOS magnetograms have a cadence of 45 seconds with a two-pixel resolution of 1 " resolution, a noise level of $17 \mathrm{G}$, and a dynamic range of $\pm 3 \mathrm{kG}$. The vector magnetograms have a 12 -minute cadence with a polarization accuracy of no less than $0.3 \%$. HMI thus provides the first rapid-cadence measurements of the strength and direction of the solar magnetic field over the visible disk of the Sun.

\section{Science Data Capture Requirement}

An important consideration during the post-launch science phase, or Phase E, is the science data-capture requirement, driven primarily by the needs of the helioseismic studies. Long, uninterrupted periods of continuous observation provide the best data for the helioseismology algorithms that are used to study the internal structure of the convection zone. The development of predictive models for short-term phenomena such as flares and CMEs also relies on a low-latency, continuous dataset. SDO is designed to return science data for at least 22 individual 72-day periods over the five-year mission. For one 72-day period to be considered complete, the ground system must capture $95 \%$ of all possible observation time. The remaining 5\% is covered in the SDO Science Data Capture Budget, which accounts for science-data outages and interruptions expected during the mission. These outages and interruptions include:

- Planned operations, such as thruster-based, station-keeping maneuvers, and high-gain antenna (HGA) handovers. SDO uses thrusters to maintain the longitude and inclination of its orbit and to control the momentum in the reaction wheels. HGA handovers occur near eclipses when a single antenna cannot maintain contact with SDOGS while keeping the observatory oriented along the solar-rotation axis (solar North).

- Instrument calibration, roll, and off-point maneuvers. Each instrument has a set of calibration maneuvers that are performed on a regular basis. Some, such as daily flatfields and internal calibrations, affect only a single instrument. Others, such as roll maneuvers, affect all of the instruments.

- Eclipses of the Sun by the Earth and transits of the Moon across the Sun. For several weeks around the Equinoxes, SDO will experience eclipses when the Earth passes between the satellite and the Sun. This causes a loss of 44 hours each year. Lunar transits 
occur at least once per year and a maximum of four times a year during the SDO mission. Transits of Mercury (on 9 May 2016 and 11 November 2019) and Venus (on 6 June 2012) across the solar disks are counted toward good science data as they are used for calibration purposes (100 hours per year).

- Random interruptions caused by energetic charged particles (Single Event Upsets and Solar Energetic Electrons: 34 hours per year).

- Unplanned interruptions at SDOGS include signal attenuation due to rain, interference of direct solar radio emissions when SDO is directly in line with the Sun and the ground station, and equipment problems (112.5 hours per year).

Whenever a maneuver moves the instruments off of the Sun, it is necessary to include time for thermal and alignment recovery.

The Flight Operations Team (FOT) and instrument operations teams are jointly responsible for tracking science data losses. Planned events causing data loss will be scheduled in order to satisfy the requirements for data capture and completeness. For data to be considered complete, the ground system must forward sufficient downlinked HMI data to the JSOC to allow HMI to construct $95 \%$ of anticipated Dopplergrams with $99 \%$ of the pixels containing science data. The AIA and EVE instruments have less stringent data-capture requirements of $90 \%$. In the event that more than 5\% (HMI) or 10\% (AIA and EVE) of the data are lost before a 72-day period is completed, the PIs may jointly elect to start a new 72-day period during which the data-capture requirement will be enforced. After one year of science operations, we have completed five 72-day observation periods without an interruption. As of 1 April 2011 the ground system is forwarding $99.97 \%$ of the transmitted Instrument Multiplexing Protocol Data Units (IM_PDUs) to the Science Operation Centers (SOCs) and HMI has recovered $97 \%$ of the Dopplergrams and magnetograms. As anticipated, the eclipse seasons suffer from the greatest loss of data.

\subsection{Ground System and Mission Operations}

SDO uses a dedicated ground station with dual antennas to meet science-data downlink completeness requirements. The ground system can receive a science-data downlink rate of 130 Mbps for 24 hours per day and seven days per week to achieve full mission success. Once on the ground, the science data are assembled into files and transmitted to the instrument SOCs. A local, 30-day science data cache is available at the ground station. The ground station also provides S-band frequency command, telemetry and tracking functions in support of SDO mission operations. The SDO Ground System is described by Tann, Pages, and Silva (2005).

Because the instruments view the full disk of the Sun, there is no need to have an elaborate commanding process. SDO is pointed at the center of the Sun except for calibration and spacecraft maneuvers. Thus the data are quite uniform, simplifying the data access, i.e. no campaigns. The high-duty cycle allows external users to obtain concurrent observations by "observing the database" of SDO rather than coordinating special observing sequences.

\section{SDO Orbit and Mission Phases}

The rapid cadence and continuous coverage required for SDO science observations led to placing the satellite into an inclined geosynchronous orbit. This allows for a continuous, high data rate, contact with a single, dedicated ground station. When viewed from the ground station, the orbit resembles an elongated figure-eight (or annalemma) with a width 
of $(i / 4) \sin i=3.3^{\circ}$ as it orbits the Earth once per day. Relying on a single site reduces the complexity of the ground system by removing the need to combine data from multiple, widely spaced antennas scattered around the globe.

Nearly continuous observations of the Sun can be obtained from other orbits, such as a Sun-synchronous, low-Earth orbit (LEO). If SDO had been placed into a LEO, it would have been necessary to store large volumes of scientific data onboard until a downlink opportunity was available, and multiple sites around the globe would be needed to downlink the data. However, no space-qualified data recorder with the capability to handle this large data volume exists. The large data rate of SDO, the unavailability of a data recorder, and the ability to continuously stream data from the spacecraft if a geosynchronous orbit were selected led to the selection of the inclined geosynchronous orbit.

Disadvantages of the inclined geosynchronous orbit include higher launch costs (relative to LEO) and two Earth-shadow (eclipse) seasons each year. During these two - three week long eclipse periods, SDO will experience a daily interruption of solar observations, and these interruptions have been included in SDO's data-capture budget (see Section 7). There will also be three lunar transits per year from this orbit.

This inclined geosynchronous orbit is located on the outer edges of Earth's radiation belt, where the radiation dose can be quite high. Additional shielding was added to reduce the effects of exposure to this ionizing radiation.

The combined Atlas V/Centaur launch vehicle placed SDO into a geosynchronous transfer orbit with an apogee altitude of $35350 \mathrm{~km}$ and a perigee altitude of $2500 \mathrm{~km}$. A series of nine apogee-motor burns were used to raise perigee altitude to $35350 \mathrm{~km}$, and then three thruster motor burns were used to raise both apogee and perigee altitudes to their final values of $35800 \mathrm{~km}$ (Figure 2). Excessive fuel-slosh during the early main engine burns delayed the reaching of the final orbit until 16 March 2010. Once SDO was on-orbit the main engine was disabled, leaving only the thrusters to perform maneuvers.

Two types of on-orbit maneuver are used. One maneuver changes the spacecraft velocity, known as a delta-V maneuver, and is used to keep the spacecraft within its assigned orbit. The other maneuver, called a delta-H (where " $\mathrm{H}$ " is the symbol for angular momentum), is used to dissipate the momentum stored during regular operations. At the end of its mission, SDO will be moved to a disposal orbit outside of the geosynchronous belt and all energy sources depleted.

\section{SDO Data}

Science data from SDO are transmitted via Ka-band to the dedicated ground station in New Mexico. From there the data stream is converted into telemetry files and forwarded to the SOCs. Each instrument is assigned a fraction of the telemetry stream. Because continuous contact is maintained with the spacecraft, the science-data return is maximized.

Data from the SDO mission consist of images, full-disk maps, and spectra. Each SDO science investigation team maintains a data archive of its science data products for the life of the SDO mission, and have made these data publicly available. Given the volume of data produced by SDO, a variety of methods have been developed to obtain data at both reduced and full resolutions. The data latency for near-realtime data has been 15 minutes during the mission operations phase.

Full-resolution science data from HMI and AIA are served by the Joint-SOC (JSOC) at Stanford University (http://jsoc.stanford.edu/ajax/lookdata.html). The data are provided as FITS formatted files (a description of the FITS format can be found at Pence et al., 


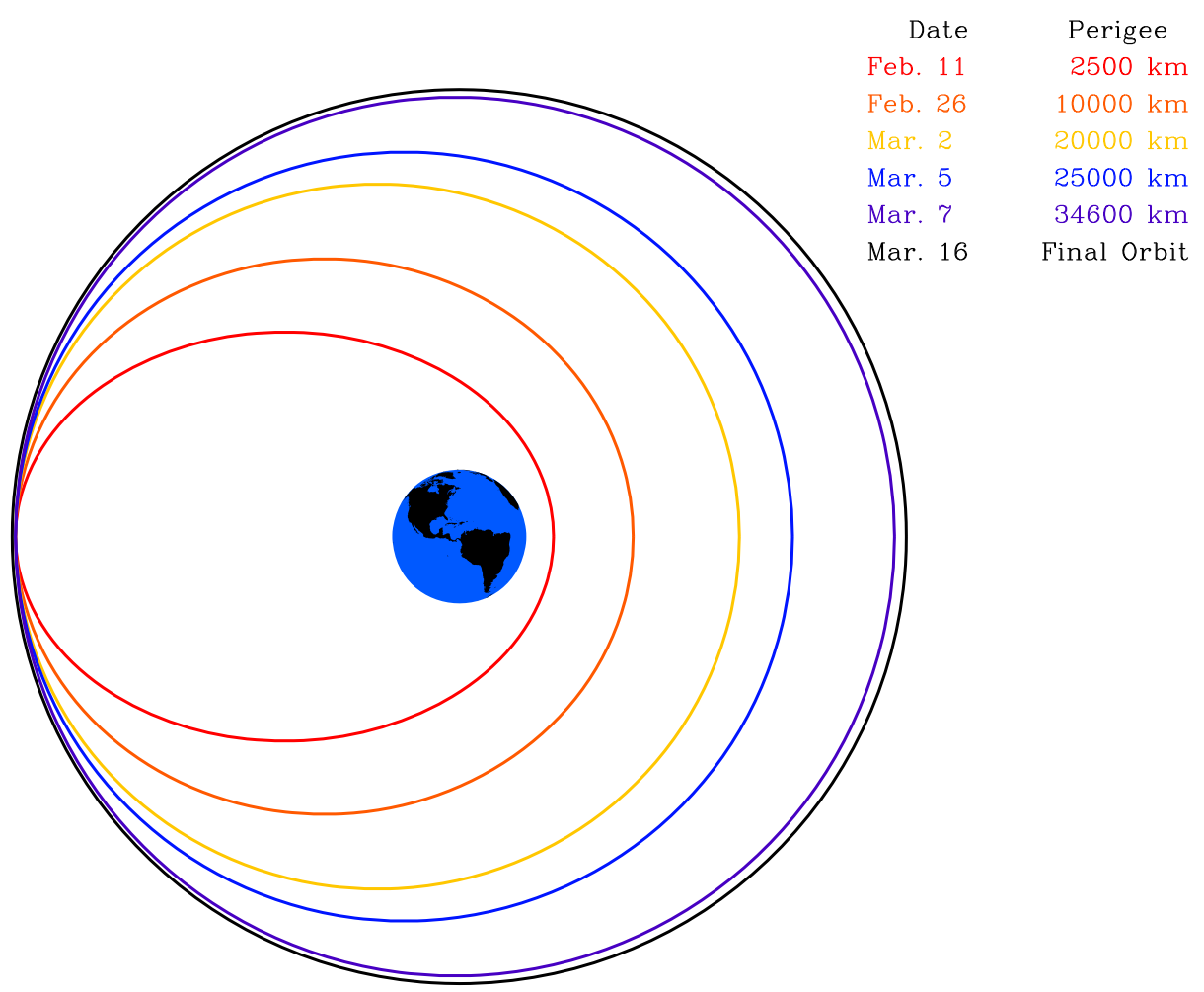

Figure 2 The raising of the SDO orbit from GTO to inclined geosynchronous. The initial GTO, two intermediate orbits, and the estimated final orbit are shown to scale. Apogee Motor Firings (AMFs) all took place at the apogee of the orbit (the left side of the diagram) and were designed to lift the perigee up to a geosynchronous altitude. A few trim maneuvers then moved SDO into its final orbit. Perigee values are altitudes (height above the Earth).

2010). Readers for files in the FITS formats for most languages can be found at http://heasarc.gsfc.nasa.gov/docs/software/fitsio/fitsio.html. The HMI full-disk maps, such as Dopplergrams and magnetograms, along with supporting images are served at Level 1.5. AIA data can be obtained as Level 1.5 images or as Level 1.0 data that must be promoted to Level 1.5 by user software. Descriptions of the AIA data-product levels can be found in Lemen et al. (2011). Routines for this purpose are available in the IDL language through the SolarSoft package (Freeland and Handy, 1998) available at http://www.Imsal.com/solarsoft/. A synoptic sequence of all AIA bandpasses in $1 \mathrm{~K} \times 1 \mathrm{~K}$ FITS files with three-minute cadence is also provided for long-term analysis.

Full-resolution science data from EVE are served by the EVE SOC at the University of Colorado (http://lasp.colorado.edu/eve/data/data_access.htm). These data are available in compressed FITS files. The EVE team also produces daily averaged data at three resolutions. The EVE team provides a FITS reader routine in the IDL language that can be used to read their data files. 


\subsection{Rules of the Road for Data Use}

The SDO science investigators agree to abide by the "Rules of the Road" developed for the Sun-Earth Connection and its successor, the Heliophysics Science Division. These rules are available at http://lwsde.gsfc.nasa.gov/Rules_Revised20030327.html and repeated here:

i) The Principal Investigators (PI) shall, in a timely manner, make available to the science-data user community (Users) data and access methods to reach the scientifically useful data and provide analysis tools equivalent to the level that the PI uses.

ii) The Principal Investigators (PI) shall make available appropriate data products to the public that assist the PI's Education and Public Outreach responsibilities.

iii) The PI shall ensure that all scientifically important data are archived to ensure longterm accessibility of the data and their correct and independent usability.

iv) The PI shall notify Users of updates to processing software and calibrations via metadata and other appropriate documentation.

v) Users should consult with the PI to ensure that the Users are accessing the most recent available versions of the data and analysis routines.

vi) Browse products are not intended for science analysis or publication and should not be used for those purposes without consent of the PI.

vii) Users should acknowledge the sources of data used in all publications, presentations, and reports.

viii) Users should transmit to the PI a copy of each manuscript that uses the PI's data upon submission of that manuscript for consideration of publication. On publication the citation should be transmitted to the PI and any other providers of data.

ix) Users are encouraged to make tools of general utility and/or value-added data products widely available to the community. Users are encouraged to notify the PI of such utilities or products. The User should also clearly label the product as being different from the original PI-produced data product.

$x$ ) The editors and referees of scientific journals should avail themselves of the expertise of the PI while a data set is still unfamiliar to the community, and when it is uncertain whether authors have employed the most up-to-date data and calibrations.

\subsection{Browse and Public Access Data}

SDO data are made available to the public in common graphics formats at several websites. For example, the mission website at http://sdo.gsfc.nasa.gov/ serves images in the JPEG format at a roughly 15-minute cadence and several spatial resolutions. Movies covering the previous 48 hours are available in both mpeg format and as self-updating kiosk movies. The instrument teams provide access to similar graphics formats at more rapid cadences. Helioviewer (http://helioviewer.org/) and jHelioviewer (http://jhelioviewer.org/) allow one to browse the AIA images, making and sharing movies from an archive of images in the JPEG2000 format.

Press releases, graphics of wide interest, and other announcements are available from NASA Headquarters at http://www.nasa.gov/sdo.

\subsection{Final Data Archive}

At the end of the SDO mission, each SIT will deliver the data obtained during the prime mission to a data archive for permanent storage. 


\section{Summary}

The Solar Dynamics Observatory is setting the standard for new rapid-cadence science data that enables the study of the energetics of solar events in the corona and the internal structure and surface magnetic of the Sun. SDO data are being combined with that from other satellites in NASA Heliophysics Great Observatory, such as STEREO, SOHO, Wind, TIMED, and ACE, to develop an understanding of how solar activity is generated and how it can affect the Earth.

Many new, significant discoveries have already occurred, with more to follow as this mission progresses. In particular, researchers hope to learn how storms get started near the solar surface and how they propagate upward through the solar atmosphere toward Earth and elsewhere in the solar system. Scientists will use SDO data to help them understand how the Sun's changing magnetic fields are created and how they evolve to release the energy that heats the corona and creates the eruptions that are the storms of solar activity and the seeds of space weather.

Acknowledgements This work was supported by NASA's Solar Dynamics Observatory (SDO).

Open Access This article is distributed under the terms of the Creative Commons Attribution Noncommercial License which permits any noncommercial use, distribution, and reproduction in any medium, provided the original author(s) and source are credited.

\section{References}

Freeland, S.L., Handy, B.N.: 1998, Solar Phys. 182, 497.

Hathaway, D., Antiochos, S., Bogdan, T., Davila, J., Dere, K., Fleck, B., et al.: 2001, Solar Dyanmics Observatory: Report of the Science Definition Team. Technical Report NP-2001-12-410-GSFC, NASA. http://sdo.gsfc.nasa.gov/assets/docs/sdo_sdt_report.pdf.

Lemen, J.R., Title, A.M., Akin, D.J., Boerner, P.F., Chou, C., Drake, J.F., Duncan, D.W., Edwards, C.G., Friedlaender, F.M., Heyman, G.F., Hurlburt, N.E., Katz, N.L., Kushner, G.D., Levay, M., Lindgren, R.W., Mathur, D.P., McFeaters, E.L., Mitchell, S., Rehse, R.A., Schrijver, C.J., Springer, L.A., Stern, R.A., Tarbell, T.D., Wuelser, J.-P., Wolfson, C.J., Yanari, C.: 2011, Solar Phys. doi:10.1007/s11207-011-9776-8.

Pence, W.D., Chiappetti, L., Page, C.G., Shaw, R.A., Stobie, E.: 2010, Astron. Astrophys. 524, A42. doi:10.1051/0004-6361/201015362.

Schou, J., Scherrer, P.H., Bush, R.I., Wachter, R., Couvidat, S., Rabello-Soares, M.C., Bogart, R.S., Hoeksema, J.T., Liu, Y., Duvall Jr., T.L., Akin, D.J., Allard, B.A., Miles, J.W., Rairden, R., Shine, R.A., Tarbell, T.D., Title, A.M., Wolfson, C.J., Elmore, D.F., Norton, A.A., Tomczyk, S.: 2011, Solar Phys. doi:10.1007/s11207-011-9842-2.

Schrijver, C.J., Title, A.M.: 2011, J. Geophys. Res. 116, A04108. doi:10.1029/2010JA016224.

Solanki, S.K., Usoskin, I.G., Kromer, B., Schüssler, M., Beer, J.: 2004, Nature 431, 1084. doi:10.1038/ nature02995.

Tann, H.K., Pages, R.J., Silva, C.J.: 2005, In: Space Systems Engineering Conference GT-SSEC.C.5. http://hdl.handle.net/1853/8029.

Woods, T.N., Eparvier, F.G., Bailey, S.M., Chamberlin, P.C., Lean, J., Rottman, G.J., Solomon, S.C., Tobiska, W.K., Woodraska, D.L.: 2005, J. Geophys. Res. 110, A01312. doi:10.1029/2004JA010765.

Woods, T.N., Eparvier, F.G., Hock, R., Jones, A.R., Woodraska, D., Judge, D., Didkovsky, L., Lean, J., Mariska, J., Warren, H., McMullin, D., Chamberlin, P., Berthiaume, G., Bailey, S., Fuller-Rowell, T., Sojka, J., Tobiska, W.K., Viereck, R.: 2011a, Solar Phys. doi:10.1007/s11207-009-9487-6.

Woods, T.N., Hock, R., Eparvier, F., Jones, A.R., Chamberlin, P.C., Klimchuk, J.A., Didkovsky, L., Judge, D., Mariska, J., Warren, H., Schrijver, C.J., Webb, D.F., Bailey, S., Tobiska, W.K.: 2011b, Astrophys. J. 739, 59. doi:10.1088/0004-637X/739/2/59. 